\title{
Adjunctive bone grafting in arthroscopic surgery for symptomatic meniscal tearing
}

Patel M. Mihir*, Shah Kishan, Patel M. Sahvan, Patel S. Sia and Hillenburg John

*Correspondence: mpatel@orthoindy.com

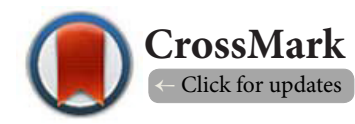

Ortholndy Hospital, USA.

\begin{abstract}
Meniscal tears are prevalent throughout the population. These tears can become symptomatic for patients including locking, giving way, and buckling, creating instability. Conservative treatment measures initially prior to instability developing may include physical therapy, alternative shots, and rest. Patients may develop bone edema in the proximal tibia following meniscal tearing which may warrant further index treatment measures and a modified physical therapy program when compared to those patients that do not have any bone edema.
\end{abstract}

Keywords: Meniscal tear, Bone marrow lesion, Osteochondritis dissecans, Stress fracture, Stress reaction, Knee instability

\section{Introduction}

Meniscal tears are prevalent throughout the population. These tears can become symptomatic for patients including locking, giving way, and buckling, creating instability. Conservative treatment measures initially prior to instability developing may include physical therapy, alternative shots, and rest. Patients may develop bone edema in the proximal tibia following meniscal tearing which may warrant further index treatment measures and a modified physical therapy program when compared to those patients that do not have any bone edema.

\section{Case presentation}

\section{Basic Science}

With weight bearing, the menisci transfer forces outwards away from the tibia. As menisci tear, the forces may increase across the proximal tibia. As menisci develop complex tears, patients may develop instability as well, creating additional force across the proximal tibia. The proximal tibia may not have the capacity to handle the excess force, develop bone edema, and possibly a stress fracture. Treatment methods include initially non weight bearing with assistive devices, bracing, and physical therapy. When these measures fail and instability develops, additional arthroscopic treatment of the menisci may be beneficial with bone grafting of the proximal tibia.

\section{Materials and methods}

From 2014 to 2016, 42 patients had symptomatic instability with stress reactions and meniscal tearing documented on MRI with no greater than Grade II arthritic changes on plain weight bearing $x$-rays. Following failed conservative treatment measures, arthroscopic outpatient treatment of the menisci with adjunctive bone grafting with auto graft and 5 cc of Cerament (Bonesupport, Inc) of the proximal tibia took place. Postoperatively the patients were seen 1-2 days following the procedure and their dressing changed. Partial weight bearing was recommended along with physical therapy to start the following week. Patients followed up 2 weeks later, sutures were removed, and $x$-rays obtained. The patients then followed up monthly with $x$-rays of the knee to assess bone graft incorporation until their symptoms resolved and were asked to follow up as needed.

\section{Results}

Twenty two females and 20 males underwent the procedure. Forty seven procedures were done. Five patients had staged bilateral procedures done. Average age was 53. Average follow up was 14 months. No infections occurred. Preoperative average HSS knee score was 53. Postoperative HSS knee score was 92. ( $p$ value of 0.01 ). The five staged bilateral procedures were 
done between three to five months after the index operation. All 42 had bone graft incorporated with no nonunions or malunions at the harvest site. None of the 47 procedures developed bone edema postoperatively.

\section{Discussion}

Percutaneous subchondroplasty has been described for isolated bone edema lesions in the knee with excellent results. Davis et al showed that subchondroplasty is an effective treatment modality for knee osteoarthritis and bone marrow edema, hopefully delaying the need for a total knee replacement. In patients with meniscal tears and bone edema, our results indicate patients would benefit from adjunctive bone grafting at the edema site with concomitant arthroscopic treatment of the meniscus tears to help the stress reaction and advance physical therapy postoperatively. Cohen et al described that isolated arthroscopic debridement beyond 6 months for bone marrow lesions was not effective but subchondroplasty for osteoarthritis with bone marrow lesions may be a promising approach. Byrd et al showed that midterm outcomes for subchondroplasty $95 \%$ would undergo subchondroplasty again and $96 \%$ would recommend the procedure. Twenty five percent went on to total knee arthroplasty.

Isolated meniscal tears may not necessarily need adjunctive bone grafting. Yoo et al described no early compromise of a total knee in patients who underwent total knee arthroplasty following subchondroplasty.

\section{Conclusion}

Adjunctive bone grafting for symptomatic meniscal tearing with concomitant bone marrow edema in the proximal tibia appears to be providing pain relief and improving functional outcomes in short and midterm follow up for patients.

\section{Competing interests}

The authors declare that they have no competing interests.

Authors' contributions

\begin{tabular}{|l|c|c|c|c|c|}
\hline Authors' contributions & MMP & KMS & SMP & SSP & JH \\
\hline Research concept and design & $\checkmark$ & $\checkmark$ & $\checkmark$ & $\checkmark$ & $\checkmark$ \\
\hline Collection and/or assembly of data & $\checkmark$ & $\checkmark$ & $\checkmark$ & $\checkmark$ & $\checkmark$ \\
\hline Data analysis and interpretation & $\checkmark$ & $\checkmark$ & $\checkmark$ & $\checkmark$ & $\checkmark$ \\
\hline Writing the article & $\checkmark$ & $\checkmark$ & $\checkmark$ & $\checkmark$ & $\checkmark$ \\
\hline Critical revision of the article & $\checkmark$ & $\checkmark$ & $\checkmark$ & $\checkmark$ & $\checkmark$ \\
\hline Final approval of article & $\checkmark$ & $\checkmark$ & $\checkmark$ & $\checkmark$ & $\checkmark$ \\
\hline Statistical analysis & $\checkmark$ & $\checkmark$ & $\checkmark$ & $\checkmark$ & $\checkmark$ \\
\hline
\end{tabular}

Publication history

Editor: Antonio G Tristano, Medical Center Carpetana, Spain.

Received: 19-Nov-2017 Final Revised: 05-Jan-2018

Accepted: 08-Jan-2018 Published: 26-Jan-2018

\section{References}

1. Adrian Thomas Davis, Jennifer Marie Byrd, Justin Angelo Zenner, Darren A. Frank, Patrick J. DeMeo and Sam Akhavan. Short-Term Outcomes of the Subchondroplasty Procedure for the Treatment of Bone Marrow Edema Lesions in Patients with Knee Osteoarthritis. Orthopaedic Journal of Sports Medicine. 2015.

2. Cohen SB and Sharkey PF. Subchondroplasty for Treating Bone Marrow Lesions. J Knee Surg. 2016; 29:555-563. | Article | PubMed

3. Jennifer Marie Byrd, Sam Akhavan and Darren A. Frank. Mid-term Outcomes of the Subchondroplasty Procedure for Patients with Osteoarthritis and Bone Marrow Edema. Orthopaedic Journal of Sports Medicine. 2017.

4. Yoo JY, O'Malley MJ, Matsen Ko LJ, Cohen SB and Sharkey PF. Knee Arthroplasty After Subchondroplasty: Early Results, Complications, and Technical Challenges. J Arthroplasty. 2016; 31:2188-92. | Article | PubMed

\section{Citation:}

Mihir PM, Kishan S, Sahvan PM, Sia PS and John $\mathrm{H}$. Adjunctive bone grafting in arthroscopic surgery for symptomatic meniscal tearing.

$J$ Rheumatol Orthop. 2018; 5:1. http://dx.doi.org/10.7243/2055-7000-5-1 